\title{
Image Segmentation by Means of Fuzzy Entropy Measure
}

\author{
C. Di Ruberto, M. Nappi, S. Vitulano \\ Istituto di Medicina Interna - Policlinico Universitario \\ Via S. Giorgio, 12 - 09124 Cagliari - ITALY \\ tel. (39) (70) 6028209 - fax (39) (70) 663651 \\ E-Mail vitulano@vaxca1.unica.it
}

\begin{abstract}
The paper describes an algorithm for image segmentation using fuzzy entropy measure. The relation between the fuzzy entropy of an image domain and the fuzzy entropy of its subdomains is explored as a uniformity predicate. With the aim of implementing the model, we have introduced a well known technique of Problem Solving. The most important roles of our model are played by the Evaluation Function $(E F)$ and the Control Strategy. So the $E F$ is related to the ratio between the fuzzy entropy of one region or zone of the picture and the fuzzy entropy of the entire picture. The Control Strategy determines the optimal path in the search tree (quadtree) so that the nodes of the optimal path have minimal fuzzy entropy. The paper shows some comparisons between the proposed algorithm and classical edge detection techniques.
\end{abstract}

\section{INTRODUCTION}

Segmentation is a significant issue in the field of image processing and image understanding. The segmentation is the process, both human and automatic, that isolates in a pictorial scene zones or regions, edges or contours and angles with respect to a certain uniformity predicate.

In the last years several approaches have been proposed in the literature and may be classified as follows:

- Local (or atomistic) approach:

Such a method takes advantages of grey level discontinuities that are considered relevant features of image. In order to extract these features several local operators have been introduced and the most important are Sobel [1, 2], Marr-Hildreth [3]. In this case the discontinuities assume the shape of monodimensional step function. In [1] Gonzales and Wintz propose an effective algorithm that looks at regions in image with non-unimodal histogram, using local thresholds.

To the aim of resolving the strong simplification that associates edges with a monodimensional step function, a lot of corrections have been introduced. In this manner local operators can process image with smoothing and shading effects $[2,4,5]$. In $[6,7]$ any angle of a region in a digital image is approximated by means of a series of segments or half edges, where a half edge can be characterised by its position and orientation angle. So the angle is a point at which two (or more) half edges cross [7-9]. 
Recently some techniques based on image iterated smoothing have been proposed. Such a method eliminates higher frequencies of image by iterated sampling, preserving -as much as possible- the shape and the edge positions $[10,11]$. This approach presents some obvious limits such as an high computing time, and the strictly depending on threshold and on smoothing parameters. For this reason usually local operators are not utilised to process medical images.

In [12] Higgins presents and compares three different methods to detect grey level discontinuities by utilising structural local informations.

- Global (or structural) approach:

For its simplicity the global threshold is the oldest technique for image segmentation. In this approach it is customary to utilise one threshold for the whole image (global information) or one threshold for each image region (contextual method) [13-16]. The threshold is based on the following rules: each region in image may be associated with a peak histogram [17-20]. Of course such a rule is very restrictive.

In order to extract the objects that make up an image, entropy -high order entropy or conditional entropy - is often adopted as uniformity predicate [21,22].

The Gestalt theory and Neurophysiologic theory affirm that in the human perceptive process the eye aims to minimise objects and background variations -homogeniseenhancing transitions regions, i.e. edges.

The goal of this paper is to propose a new algorithm for image segmentation using the fuzzy entropy, namely FISE, exploiting the ratio between each image region and the whole image $[23,24,32]$. In other words such a ratio is minimum on the regions and maximum on the edges.

\section{Fuzzy ENTRopy MeAsure for EdGe Detectiox}

The task of the segmentation is to enhance the different regions of the image. In order to segment the image into regions each of them has to satisfy the uniformity predicate. Now it is necessary to analyse some aspects of the uniformity predicate before we introduce it.

In our opinion the uniformity predicate couldn't be determined without considering the features of the whole image. In other words the measures calculated to determine the uniformity predicate have to be related to the same measures calculated on the whole image. In this paper the fuzzy entropy has been chosen as the uniformity predicate. In particular the fuzzy entropy of a region (or a background) -that is a region too- is always lower than the entropy of whole image or, in other words, the fuzzy entropy of a region is always greater or equal than the entropy of its subdomains.

The FISE adopts a strategy to segment the image similar to that introduced in [25]: the merge and the split are applied in cascade on the image using a region growing algorithm.

A search tree of any hierarchical structure, as for example t-ari trees, can be utilised. In our work we have chosen the quadtree.

The strateg: we'll propose segments into regions whose fuzzy entropy is nearly zero. More precisely, the zones extracted as regions are those whose evaluation function is gradually goes to zero.

We have defined the evaluation function as a measure of the fuzzy entropy of a region according to the theorem of fuzzy entropy [33]. 
The theorem of fuzzy entropy defines the entropy of a fuzzy set $A$ as a measure of the subset $A$ and not $A$ ( $A$ and $A^{c}$ ) in relation to the subset $A$ or not $A$ ( $A$ or $A^{c}$ ) as showed in Figure 1.

Let $a_{i}$ be an internal node of quadtree whose grey tones are $g_{1}, g_{2}, \ldots, g_{n}\left(g_{i}<g_{j}\right.$, $i<j, i, j=1, \ldots, n)$ and frequencies $f_{1}, f_{2}, \ldots, f_{n}$, respectively.

Let $a_{4 i+t}, t \in[1,4]$, a child node of $a_{i}$, whose greytones are $g_{1}^{\prime}, g_{2}^{\prime}, \ldots, g_{m}^{\prime}$ $\left(g_{i}^{\prime}<g_{j}^{\prime}, i<j, i, j=1, \ldots, m\right)$ and frequencies $f_{1}^{\prime}, f_{2}^{\prime}, \ldots, f_{m}^{\prime}$, respectively.

For each grey tone $g_{i}, i=1, \ldots, n$, of the node $a_{i}$ we define

$$
\Delta f_{i}=\left|f_{i}-f^{\prime}\right|
$$

(00)

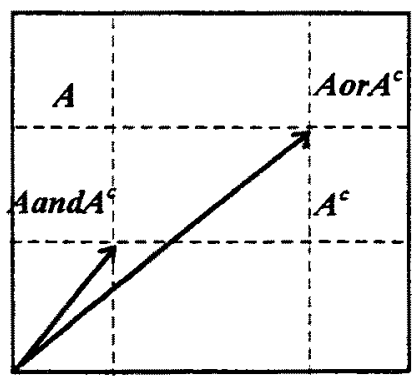

Figure 1 - The entropy fuzzy theorem.

where $f_{i}$ is the frequency of the grey tone $g_{i}$ while $f_{j}^{\prime}$ is the frequency of the grey tone of the node $a_{4 i+t}$, with $g_{i}=g_{j}^{\prime}$.

Then we evaluate

$$
\Delta^{100} f_{i}=\sqrt{\left(100-\Delta f_{i}\right)^{2}+100^{2}}
$$

and finally we define the fuzzy entropy of the grevtone $g_{i}$ as:

$$
e_{i}=\frac{\Delta f_{i}}{\Delta{ }^{100} f_{i}} \text {. }
$$

So, the fuzzy similarity of the node $a_{i}$ respect to its child node $a_{4 i+t}$ is:

where $K=\max (n, m)$.

$$
E\left(a_{i}, a_{4 i+t}\right)=\frac{1}{K} \sum_{i=1}^{K} e_{i}
$$

Now we can define the fuzzy homogeneity of the node $a_{4 i+t}, t \in[1,4$ as the average fuzzy similarity of $a_{4+t}$ and its four children nodes:

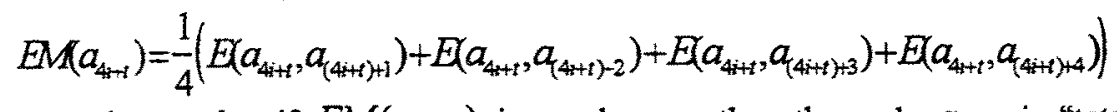

We can observe that if $\operatorname{EM}\left(a_{4 i+t}\right)$ is nearly zero then the node $a_{4 i+t}$ is "totally homogeneous". 
So, the evaluation function of the node $a_{4 i+1}$ is given by:

$$
F\left(a_{4 i+t}\right)=\frac{E M\left(a_{41+t}\right)}{E\left(a_{i}, a_{4 i+t}\right)} .
$$

$F\left(a_{4 i+1}\right)$ evaluates the fuzzy homogeneity of the node $a_{4 i+t}$ respect to the fuzzy similarity to its father node $a_{i}$, assuming minimal value inside a region and maximal value along the contour of a region.

So, if $a_{3}$ is an internal node of a quadtree and the evaluation functions of its four children nodes are $F\left(a_{4 i+1}\right), F\left(a_{4 i+2}\right), F\left(a_{4 t-3}\right), F\left(a_{4 i+4}\right)$, the control strategy expands the child node $a_{s}$, with $s=4 i+1, \ldots, 4 i+4$, whose $F\left(a_{s}\right)$ is minimal:

$$
F\left(a_{s}\right)=\min \left\{F\left(a_{4 i+1}\right), F\left(a_{4 i+2}\right), F\left(a_{4 i+3}\right), F\left(a_{4 i+4}\right)\right\} \text {. }
$$

According this rule, along the search path of the quadtree, $F\left(a_{s}\right)=\frac{E M\left(a_{s}\right)}{E\left(a_{i}, a_{s}\right)}$ is different from zero, in particular $E M\left(a_{s}\right) \neq 0$ and $E\left(a_{i}, a_{s}\right) \neq 0$.

Applying this rule at each level of the search tree, we'll individuate a node $a$, whose $E M\left(a_{j}\right) \cong 0$ and so $F\left(a_{j}\right) \cong 0$. Then we can state that the subdomain relative to the node $a$, belongs to a region (or an object) $R$.

If, inside the region $R$ the evaluation function $F\left(a_{i^{\prime}}\right)$ of an expanded node $a_{i^{\prime}}$ is nearly zero and the evaluation function $F\left(a_{j}\right)$ of its child node $a_{j^{\prime}}$ to expand is different from zero then we can state that the subdomain relative to the node $a_{i}$ is the smallest subdomain including the partition element of the region. In other words, if the dimension of the subdomain relative to the node $a_{i^{\prime}}$ is $k_{i^{\prime}} \times k_{i^{\prime}}$ and its initial coordinates in the entire image domain are $\left(x_{i}, y_{i}\right)$, then the dimension of the partition element $P$ of the region $R$ will be $d x \times d y$, with $k_{i^{\prime} / 2} \leq d x \leq k_{i^{\prime}}$, $k_{i^{\prime} / 2} \leq d y \leq k_{i^{\prime}}$ and $d x=\min \{x\}, k_{i^{\prime} / 2} \leq x \leq k_{i^{\prime}}$ and $d y=\min \{y\}, k_{i^{\prime} / 2} \leq y \leq k_{i^{\prime}}$ such that the evaluation function computed on the subdomain with dimension $x \times y$, included in the domain of the node $a_{i^{\prime}}$, and initial coordinates $\left(x_{i^{\prime}}, y_{i^{\prime}}\right)$ is nearly zero. So, the region $R$ of the image will be the union of the elements (or subdomains) $P_{1}, P_{2}, \ldots, P_{N}$ fuzzy similar to the partition element $P$ :

$$
R=\bigcup_{i=1}^{N} P_{i}
$$

where $P_{i}$ has coordinates $\left(p x_{i}, p y_{1}\right)$ and dimension $d x_{i} \times d y_{i}$, with $d x_{i}=d x$ and $d y_{1}=d y$.

\section{EXPERIMENTAL RESULTS AND DISCUSSIONS}

In order to evaluate the performance of the proposed edge detection algorithm we have tested it on several theoretical and real $512 \times 512 \times 8$ bits images. For the sake of brevity in this Section objective results obtained on theoretical images are presented. Moreover, FISE has been widely compared with the segmentation algorithm based on entropy (namely ISE) proposed by Vitulano et alt. in [32] and some of the most useful local 
operators, i.e. Sobel, DOG zero-crossing, Haralick [1, 2], Anisotropic diffusion and three methods proposed by Higgins [12].

We describe briefly ISE segmentation method.

Let $a_{s}$ be a node at level $z$ of quadtree and $a_{4 s-t}, t \in[1,4]$ a child node of $a_{s}$. Let $f_{k}\left(a_{s}\right)$ with $k \in[1, n]$ be the maxima frequencies of the histogram of the image area relative to the node $a_{s}$ and $f\left(a_{4 s+t}\right)$ with $l \in[1, m]$ be the maxima frequencies of the histogram of the image area relative to the node $a_{4 s+t}$.

For simplicity we denote the set of these frequencies with $\left\{f_{k}\right\}$ and $\{f\}$. With each maximum frequency $f_{i}$ in $\left\{f_{k}\right\}$ (or in $\left\{f_{l}\right\}$ ) we associate a gaussian function whose standard deviation is defined as:

$$
\sigma_{i}=\frac{1}{\sqrt{2 \pi \pi_{i}}}
$$

So, with each frequency $f_{i}$ in $\left\{f_{k}\right\}$ (or in $\left\{f_{l}\right\}$ ) we can associate an interval of graytones of the histogram of $a_{s}$ (or of: $a_{4 s+1}$ ):

$$
\left[g_{i}-\sigma_{i}, g_{i}+\sigma_{i}\right]
$$

where $g_{i}$ is the greytone with frequency $f_{i}$ and standard deviation $\sigma_{i}$ defined as in (2). If $g_{i}$ is the graytone with frequency $f_{i} \in\left\{f_{k}\right\}$ and $g_{j}$ is the graytone with frequency $f_{j} \in\left\{f_{l}\right\}$ then the following relations could be satisfied:

$$
\left|g_{i}-g_{j}\right| \leq|a-b| \leq 2 \sigma *
$$

where $a$ and $b$ are the extrema of the intersection interval $\left[g_{1}-\sigma_{1}, g_{i}+\sigma_{i}\right] \square$ $\left[g_{j}-\sigma_{j}, g_{j}+\sigma_{j}\right]$ and $\sigma^{*}=\min \left(\sigma_{i}, \sigma_{j}\right)$,

otherwise

$\left[g_{i}-\sigma_{i}, g_{i}+\sigma_{i}\right]\left[g_{j}-\sigma_{j}, g_{j}+\sigma_{j}\right]=\varnothing$

We consider now the sets $\left\{f_{k}\right\}$ and $\left\{f_{i}\right\}$ sorted according increasing graytones and we state that if $n=m$ and $\left|g_{i}-g_{j}\right| \leq 2 \sigma^{*}$, where $g_{i}$ is the greytone with frequency $f_{i} \in\left\{f_{k}\right\}$ and standard deviation $\sigma_{i}$ and $g_{j}$ is the greytone with frequency $f_{j} \in\{f\}$ and standard deviation $\sigma_{j}$, and $\sigma^{*}=\min \left(\sigma_{i}, \sigma_{j}\right)$, then the histograms of $a_{s}$ and $a_{4 s+t}$, $t \in[1,4]$, have the maxima frequencies in correspondence to the same graytones. In terms of multivariate analysis the histograms have the same variables. Moreover, if

$$
\forall i=j \in[1, n], f_{i}=f_{j} \text { and }\left|g_{i}-g_{j}\right|=0
$$

then the histograms of $a_{s}$ and $a_{4 s+t}$ have the same graytones distribution. 
If (3) and (4) are satisfied we define the domain relative to $a_{s}$ "totally homogeneous" to the domain relative to $a_{4 s+t}$.

Obviously, the relations (3) and (4) are valid for theoric images and for a limited number of nodes of the quadtree. Instead for real images it's necessary the introduction of an evaluation function to determine the homogeneity degree of two different domains.

The control strategy prefers the nodes with minimum E.F. along the search tree as in FISE method.

So, for each $f_{l} \in\left\{f_{k}\right\}$ and $f_{j} \in\left\{f_{l}\right\}$ satisfying the relation (3), we define the entropy $e_{i}$ relative to the frequencies $f_{i}$ and $f_{j}$ as:

$$
e_{i}=\frac{|a-b|}{\left|a^{\prime}-b^{\prime}\right|}
$$

where $a$ and $b$ are the extrema of the intersection interval $\left[g_{i}-\sigma_{i}, g_{i}+\sigma_{i}\right] \cap\left[g_{j}-\sigma_{j}, g_{j}+\sigma_{j}\right], a^{\prime}$ and $b^{\prime}$ are the extrema of the union interval $\left[g_{i}-\sigma_{i}, g_{i}+\sigma_{i}\right] \cup\left[g_{j}-\sigma_{j}, g_{j}+\sigma_{j}\right]$ and $g_{i}, \sigma_{i}, g_{j}$ and $\sigma_{j}$ are defined as in (3). So, we define the E.F. of $a_{s}$ in relation to $a_{4 s+t}$ as:

$$
E\left(a_{s}, a_{4 s+t}\right)=\frac{1}{p}\left(\sum_{i=1}^{p}\left(1-e_{i}\right)+\alpha\right)
$$

where $a$ is the number of frequencies of $\left\{f_{k}\right\}$ and $\{f\}$ not satisfying the relation (3) and $p=\frac{n+m-\alpha}{2}$.

For the test cases we assume the image $f$ is made up of disjoint constant intensity region corrupted by additive Gaussian noise (AWGN); i.e. if point $(x, y) \in$ region $R_{\mathrm{k}}$, than $f(x, y)=\mu_{\mathrm{k}}+\eta_{\mathrm{k}}(x, y)$, where $\mu_{\mathrm{k}}$ is the constant intensity value for points in $R_{\mathrm{k}}$ and $\eta_{\mathrm{k}}(x, y)$ is a sample of AWGN having statistics $N\left(0, \sigma_{k}{ }^{2}\right)$.

In order to determine an objective parameter to test the quality of processed images, as proposed by Haralick [31], we use two performance metrics to compare the various methods: $P(A E T E)$ and $P(T E A E)$. The first metric is the conditionally probability of a point being assigned as an edge point, given that the point is a true edge point, while the second one the conditional probability of a point being a true edge point, given that the point is assigned as an edge point. Assigned edge points are those points that a particular edge detection method assigns. True edge points are defined to be the points within the two-point wide region in which each point is adjacent to some point having a value different from it on the uncorrupted checkerboard. For each method, except for our algorithm, a threshold is adjusted until $P(A E T E) \approx P(T E A E)$. This equalisation in practice represents an even better trade-off between detecting true edge points and rejecting non-edge points.

The numerical comparison results proposed by Higgins [12], ISE and FISE are illustrated in Table 1.

Table 2 presents the numerical results obtained by applying ISE and FISE on a checkerboard image corrupted by AWGN, mean $=0$ and standard deviation $=60$. 


\begin{tabular}{|c|c|c|c|}
\hline & $P(T E A E)$ & $P(A E T E)$ & Average \\
\hline Sobel gradient & 0.660 & 0.656 & 0.658 \\
\hline DOG & 0.865 & 0.833 & 0.849 \\
\hline Haralick operator & 0.760 & 0.759 & 0.759 \\
\hline Anisotropic Diffusion & 0.889 & 0.898 & 0.894 \\
\hline Higgins-Method 1 & 0.948 & 0.920 & 0.934 \\
\hline Higgins-Method 2 & 0.828 & 0.823 & 0.825 \\
\hline Higgins-Method 3 & 0.866 & 0.847 & 0.857 \\
\hline FISE Method & $\mathbf{0 . 4 5 7}$ & $\mathbf{0 . 5 9 8}$ & $\mathbf{0 . 5 2 8}$ \\
\hline ISE Method & $\mathbf{0 . 9 7 4}$ & $\mathbf{1 . 0 0 0}$ & $\mathbf{0 . 9 8 7}$ \\
\hline
\end{tabular}

Table 1 - Results for noisy checkerboard using local structure operators, ISE and FISE.

\begin{tabular}{|c|c|c|c|}
\hline & $P(T E A E)$ & $P(A E T E)$ & Average \\
\hline FISE Method & 0.579 & 0.518 & 0.549 \\
\hline ISE Method & 0.669 & 0.956 & 0.812 \\
\hline
\end{tabular}

Table 2 - Results for noisy checkerboard using ISE and FISE methods.

\section{CONCLUDing REMARKS}

The FISE method seems that it should be helpful both for edge detection and segmentation of regions.

The most important features of such method could be summarised as follows:

- $\quad$ high noise tolerance both for real and theoretic image;

- threshold independence;

- low computing time $(n \log n)$ where $n$ is the size of the image;

- the edges detected don't present steps usually introduced by local operators.

We underline that the method doesn't need the choice of thresholds or operators dimensions and requires short consuming time. The control strategy makes totally automatic the entire process.

The checkerboard image represents the most significant test image. Infact, since it is a theoric image we know all its edge points and the regions contained in it. The theoretic image has been corrupted by additive Gaussian noise (AWGN) in order to test the goodness of different algorithms. Local operators require high computing time ([12]) and the choice both of thresholds and of operators dimensions (i.e. DOG 21x21, DOG $69 \times 69$ ) doesn't correspond to deterministic criteria. Through a visual analysis we have observed that the contours are irregular and present many breakings. Many points that are not true edge points are assigned as edge points. In general the edge image is smaller than the input image: this decreasing depends on the dimensions of the local operator. The numerical results confirm the limits of the local operators and the goodness of the global FISE and ISE methods. We have observed that FISE method works better as the complexity of the image increases. So, in the next future we are going to improve the experimentation of the method on complex real test images. 


\section{REFEREXCES}

1. R.C. Gonzales and P. Wintz, Digital Image Processing, 2nd Edn. Addison-Wesley, Reading Mass. (1987).

2. R. Rosenfeld and A.C. Kak, Digital Picture Processing, 2nd Edn. v.2, Academic Press, N.Y. (1982).

3. D.C. Marr and E. Hildreth, "Theory of edge detection", Proc. R. Soc. London B 207, 187-217 (1980).

4. B. Born, Robot Vision, MIT Press, Cambridge, Mass. (1986).

5. J. Canny; "A computation approach to edge detection", IEEE Trans. on Pattern Analysis Mach. Intell., PAMI-8, 679-698 (1986).

6. M.A. Gennert, "Detecting half-edges and vertices in images", Proc. IEEE Int. Conf. Comput. Vision Pattern Recogn., 552-557 (1986).

7. V.S. Nalwa and T.O. Binford, "On detecting edges", IEEE Trans. on Pattern Analysis Mach. Intell., PAMI-6, 699-714 (1986).

8. M.M. Fleck, "Some defects in finite-difference edge finders", IEEE Trans. on Pattern Analysis Mach. Intell., PAMI-14, 337-345 (1992).

9. Y.G. Leclerc, "Capturing the local structure in image discontinuities in two dimensions", Proc. IEEE Int. Conf. Comput. Vision Pattern Recogn., 34-38 (1985).

10. P. Perona and J. Malik, "Scale-space and edge detection using anisotropic diffusion", IEEE Trans. on Pattern Analysis Mach. Intell., PAMI-12, $629-639$ (1990)

11. P.Saint Marc, J.Chen and G.Medioni, "Adaptive smoothing: a general tool for early vision", IEEE Trans. on Pattern Analysis Mach. Intell., PAMI-13, 514-530 (1991).

12. W.E. Higgins and C. Hsu, "Edge detection using two dimensional local structure information". Pattern Recognition, 27, 277-294 (1994).

13. S.D. Yanowitz and A.M. Brukstein, "A new method for image segmentation", Comput. Vision Graphics Image Processing, 46, 82-95 (1989).

14. T. Taxt. P.J. Flynn and A.K. Jain, "Segmentation of document images", IEEE Trans. on Pattern Analysis Mach. Intell., PAMI-11, 1322-1329 (1989).

15. T.W. Ridler and S. Calvard, "Picture thresholding using an iterative selection method", IEEE Trans. on System Man and Cybern., SMC-8, 630-632 (1978).

16. P.K. Sahoo, S. Soltani, A.K.C. Wong and Y.C. Chen, "A survey of thresholding techniques", Comput. Vision Graphics Image Processing, v.41, 233-260 (1988).

17. N.R. Pal and S.K. Pal, "Object-background segmentation using a new definition of entropy", IEE Proc., Pt, 136, 284-295 (1989).

18. N.R. Pal and D. Bhandari, "On object-background classification", Int. J. Syst. Sci., 23, 1903-1920 (1992).

19. A.S. Abutaleb, "Automatic thresholding of grey level pictures using twodimensional entropy", Comput. Vision Graphics Image Processing, 47, 22-32 (1989).

20. P.J. Burt, T.Hong and A.Rosenfeld, "Segmentation and estimation on image properties through cooperative hierarchical computation", IEEE Trans. on System Man and Cybern., v.11, n.12 (1981).

21. Y. Nakagawa and A. Rosenfeld, "Some experiments on variable thresholding", Pattern Recognition, 11, 191-204 (1979).

22. J. Kittler and J. lllingworth, "Minimum error thresholding", Pattern Recognition, $19,41-47$ (1986). 
23. S.Vitulano, C.Di Ruberto and M.Nappi, "A.I. based image segmentation", Lectures Notes in Computer Science, Proc. of Int. Conf. of Image Analysis and Processing, C. Braccini, L. De Floriani, G. Vernazza (Eds.), Springer Verlag, 974, 429-434 (1995).

24. S. Tanimoto, M. Savini and S. Vitulano, "Allocation in attention vision", Human and Machine Vision, Proc. of the Third Int. Workshop on Perception, V. Cantoni (Ed.), Plenum Press, 171-180 (1994).

25. S.L. Horowitz and T. Pavlidis, "Picture segmentation by a directed split and merge procedure", Proc of the Second Intern. Joint Conf on Pattern Recognition, pp.424-433, Copenhagen, Aug. (1974).

26. N.J. Nilsson, Principles of Artificial Intelligence, Springer Verlag (1982).

27. S.Z. Selim and M.A. Ismail, "Soft clustering of multidimensional data: a semi-fuzzy clustering approach", Pattern Recognition, 15, 559-568 (1984).

28. M.S. Kamel and S.Z. Selim, "A thresholded fuzzy k-means algorithm for semi-fuzzy clustering", Pattern Recognition, 24, 825-833 (1991).

29. Q. Zhang and R.D. Boyle, "A new clustering algorithm with multiple runs of iterative procedures", Pattern Recognition, 24, 835-848 (1991).

30. M. Nappi and S. Vitulano, "A new more efficient k-means algorithm for clustering", Proc. of the Thirteenth IASTED Int. Conf., Applied Informatics, ed. M. H. Hamza, pp. 324-327 (1995).

31. R.M. Haralick, "Digital step edges from zero crossing of second directional derivatives", IEEE Trans. on Pattern Analysis Mach. Intell., PAMI-6, 58-68 (1984).

32. S. Vitulano, C. Di Ruberto, M. Nappi, "Biomedical Image Processing", Proc. III IEEE ICECS '96, Oct. 13-16, Rodos, v.2, pp.1116-1119 (1996).

33. B.Kosko, "Fuzzy Thinking: the New Science of Fuzzy Logic", Hyperion Ed. (1993). 